\title{
Review Article \\ Effects of Exercise on Spinal Deformities and Quality of Life in Patients with Adolescent Idiopathic Scoliosis
}

\author{
Shahnawaz Anwer, ${ }^{1,2}$ Ahmad Alghadir, ${ }^{1}$ Md. Abu Shaphe, ${ }^{3}$ and Dilshad Anwar ${ }^{4}$ \\ ${ }^{1}$ Rehabilitation Research Chair, Department of Rehabilitation Sciences, College of Applied Medical Sciences, \\ King Saud University, Riyadh 11433, Saudi Arabia \\ ${ }^{2}$ Dr. D. Y. Patil College of Physiotherapy, Dr. D. Y. Patil Vidyapeeth, Pune, India \\ ${ }^{3}$ Department of Physiotherapy, College of Applied Medical Sciences, Jazan University, Saudi Arabia \\ ${ }^{4}$ Department of Orthopedics, JNMC, AMU, Aligarh, India
}

Correspondence should be addressed to Shahnawaz Anwer; anwer_shahnawazphysio@rediffmail.com

Received 22 April 2015; Revised 15 June 2015; Accepted 18 June 2015

Academic Editor: Massimiliano Pau

Copyright (C) 2015 Shahnawaz Anwer et al. This is an open access article distributed under the Creative Commons Attribution License, which permits unrestricted use, distribution, and reproduction in any medium, provided the original work is properly cited.

\begin{abstract}
Objectives. This systematic review was conducted to examine the effects of exercise on spinal deformities and quality of life in patients with adolescent idiopathic scoliosis (AIS). Data Sources. Electronic databases, including PubMed, CINAHL, Embase, Scopus, Cochrane Register of Controlled Trials, PEDro, and Web of Science, were searched for research articles published from the earliest available dates up to May 31, 2015, using the key words "exercise," "postural correction," "posture," "postural curve," "Cobb's angle," "quality of life," and "spinal deformities," combined with the Medical Subject Heading "scoliosis." Study Selection. This systematic review was restricted to randomized and nonrandomized controlled trials on AIS published in English language. The quality of selected studies was assessed by the PEDro scale, the Cochrane Collaboration's tool, and the Grading of Recommendations Assessment, Development, and Evaluation System (GRADE). Data Extraction. Descriptive data were collected from each study. The outcome measures of interest were Cobb angle, trunk rotation, thoracic kyphosis, lumbar kyphosis, vertebral rotation, and quality of life. Data Synthesis. A total of 30 studies were assessed for eligibility. Six of the 9 selected studies reached high methodological quality on the PEDro scale. Meta-analysis revealed moderate-quality evidence that exercise interventions reduce the Cobb angle, angle of trunk rotation, thoracic kyphosis, and lumbar lordosis and low-quality evidence that exercise interventions reduce average lateral deviation. Meta-analysis revealed moderate-quality evidence that exercise interventions improve the quality of life. Conclusions. A supervised exercise program was superior to controls in reducing spinal deformities and improving the quality of life in patients with AIS.
\end{abstract}

\section{Introduction}

Adolescent idiopathic scoliosis (AIS) is a structural deformity of the spine with 3-dimensional deformation, including lateral shift and vertebral rotation affecting children at puberty $[1,2]$. The predisposing factors are genetic predisposition; connective tissue abnormalities; and skeletal, muscular, and neurological disturbances during growth. However, the exact cause remains unknown [3]. In the general population, the prevalence of AIS is about $2.5 \%$ with a Cobb angle of $>10$ degrees $[2,3]$. A variety of risk factors may result in higher curve progression, including female gender, age of $10-12$ years, absence of menarche, presence of thoracic curves, curve size at presentation $>25$ degrees, Risser sign of $0-1$, and residual growth potential [2-5].

The primary goal of rehabilitation for AIS is to reduce the progression of curves thereby decreasing the risk of secondary impairment, including back pain, breathing problems, and cosmetic deformities, and improve the quality of life $[3,6]$. Exercise plays a vital role in reducing curve progression and improving quality of life in AIS. Patients with thoracic curves $\leq 25$ degrees and thoracolumbar or lumbar curves $\leq 20$ degrees can effectively manage with exercise alone, whereas patients with thoracic 
curves of 25-50 degrees and thoracolumbar or lumbar curves of 20-40 degrees require bracing along with exercise $[3,7-9]$.

In a previous review, Negrini et al. (2008) reported that exercise had beneficial effects on the rate of progression and Cobb angle. They also found positive effects of exercise in reducing brace prescriptions [10]. More recently, Negrini et al. [11] reviewed the best available evidence regarding the rehabilitation approach to AIS and reported that the Society on Scoliosis Orthopaedic and Rehabilitation Treatment (SOSORT) had the best evidence-based guidelines. Low evidence was found for conservative treatment because out of 65 recommendations none had strong evidence (level I), 2 had moderate evidence (level II), and the remainder had weak evidence. Recently, Romano et al. conducted a Cochrane systematic review to evaluate the efficacy of scoliosis-specific exercise (SSE) in adolescent patients with AIS [12] and reported a lack of high-quality evidence to recommend the use of SSE for AIS. They identified one low-quality study that found the use of exercise more effective than electrostimulation, traction, and postural training to avoid scoliosis progression and one very low-quality study that found the use of SSE more effective than traditional physiotherapy for reducing brace prescriptions. Similarly, in a systematic review, Negrini et al. [13] reported lack of solid evidence for or against the effectiveness of physical exercise for reducing curve progression in AIS. In addition, Mordecai and Dabke reported low-quality evidence for the effect of exercise in the treatment of AIS [8].

To date, no systematic review has examined the effects of exercise on quality of life in patients with AIS. Therefore, more evidence is required regarding the effects of exercise on curve reduction and improvement in quality of life in AIS is required. The aim of this systematic review was to evaluate the effects of an exercise program on spinal deformities and quality of life.

\section{Methods}

2.1. Data Sources. Electronic databases, including Pubmed, CINAHL, Embase, Scopus, Cochrane Register of Controlled Trials, PEDro, and Web of Science, were searched for published studies using the keywords "postural correction," "postural curve," "Cobb angle," "quality of life," and "spinal deformities" combined with the Medical Subject Heading "scoliosis" and "exercise." The bibliographical search was restricted to randomized and nonrandomized controlled trials published in English language from the earliest available dates up to May 31, 2015 (see Search Strategy). Original authors were contacted and asked to provide the full text of potential papers that were not accessible. Two independent reviewers (Shahnawaz Anwer and Md. Abu Shaphe) selected the studies based on titles and abstracts, excluding those articles not related to the objectives of this review. Consensus between the reviewers was obtained through discussion.
Search Strategy

(1) Pubmed:

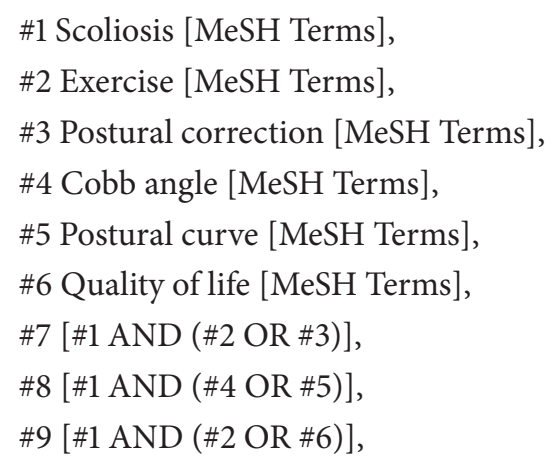

(2) Cochrane Register of Controlled Trials:

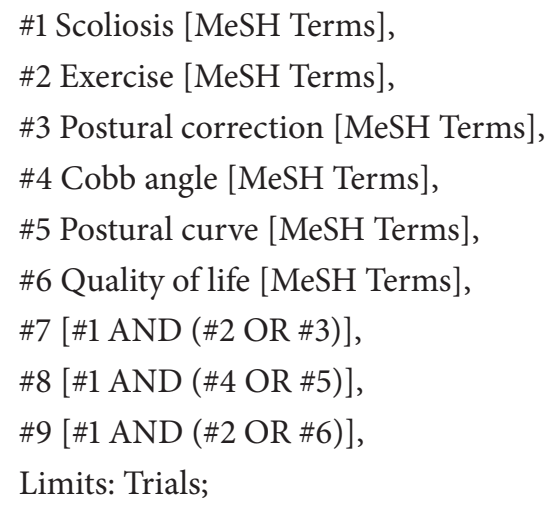

(3) PEDro:
* Advance Search,
Title or abstract: Scoliosis, exercise, Cobb angle, Quality of life,
Method: Clinical trial;

(4) Web of Science:

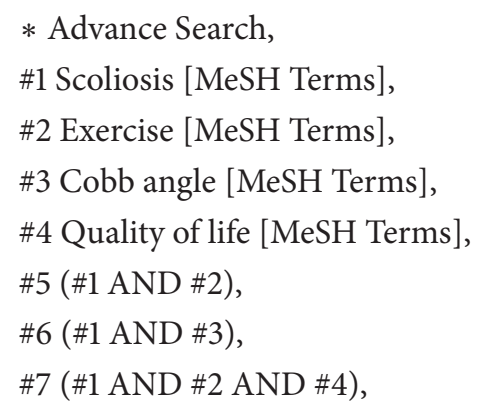


2.2. Study Selection. Studies were included on the basis of the following criteria: randomized and nonrandomized controlled methodology; subjects with AIS in the age group of 10-19 years; studies comparison of exercise with other interventions or controls; and outcome measures of radiological deformity (i.e., Cobb angle), surface deformities (including trunk rotation, thoracic kyphosis, lumbar lordosis, and average lateral deviation), and quality of life. Studies were excluded if subjects were $>19$ years of age, interventions did not include exercise or compare exercise with a control, or published results were in abstract form only. Final study selection was achieved through discussion and consensus between the two reviewers.

2.3. Data Extraction. The selected studies were screened by 2 independent reviewers (Shahnawaz Anwer and Md. Abu Shaphe). One of the reviewers had prior experience using the extraction form, systematic review methodology, and quality appraisal tools, including the PEDro and Cochrane databases, and had published two systematic reviews and a metaanalysis. The other reviewer was trained beforehand in the use of the extraction form, systematic review methodology, and quality appraisal tools, including the PEDro and Cochrane databases. The following items were extracted: author/year, design of the study, subject's characteristics, age, sex, sample size, details of exercise program (type, duration, dose, and frequency), outcomes, and conclusions. The two reviewers discussed the data with each other or consulted with a third reviewer (Ahmad Alghadir) to reach consensus. Agreement between the two reviewers was obtained using unweighted kappa $(\kappa)$ statistics. Mean and standard deviation of the baseline and final end point scores for the Cobb angle, trunk rotation, thoracic kyphosis, lumbar lordosis, lateral deviation, and function were extracted from included studies. The mean change score (final score minus baseline score) for each outcome measure was calculated for each intervention. The standardized mean difference (SMD) for all the outcomes was computed [14].

Cohen's categories were used to define the magnitude of the effect size with values of $<0.5$ as a small effect size; $\geq 0.5$ and $\leq 0.8$, as a medium effect size; and $>0.8$, as a large effect size [15]. A fixed-effects meta-analysis was conducted to determine the overall effect size of exercise. The $z$ statistic was used to test the significance of an overall effect. Cochran's $Q$ statistic and Higgins' $I^{2}$ statistic were used to determine statistical heterogeneity between studies [14]. All statistics were computed using Comprehensive Meta-Analysis software [16].

\subsection{Assessment of Methodological Quality. The 11-item PEDro} scale was used to assess the quality of included studies by two independent reviewers (Shahnawaz Anwer and Md. Abu Shaphe) [17]. A study with a score $\geq 6$ was considered highquality as reported previously [18]. In addition, the Cochrane Collaboration's tool was used to assess the risk of bias. Sequence generation, allocation concealment, blinding, completeness of outcome data, and absence of selective outcome reporting were also assessed. Risk of bias was classified as low, unclear, or high in each domain [19]. Agreement between the two reviewers in regard to the PEDro and Cochrane tools was made using unweighted kappa $(\kappa)$ statistics.

The quality of evidence was determined using the Grading of Recommendations Assessment, Development, and Evaluation System (GRADE) for each meta-analysis [20]. This method involves downgrading evidence from highquality to moderate-quality to low-quality and to very lowquality using some factors. If the majority of studies (more than $50 \%$ ) in the meta-analysis had a PEDro score $<6$ or had more than low levels of statistical heterogeneity between the studies $\left(I^{2}>25 \%\right)$ [21] or if the studies had large confidence intervals suggestive of a small number of subjects in the studies, then the evidence would be downgraded, for example, from high- to moderate-quality. In the presence of serious methodological flaws, for example, if all studies in the meta-analysis had low PEDro scores $(<6)$ with no allocation concealment and blinding, the evidence would be double downgraded (e.g., from high- to low-quality). The criteria for the grade applied to each meta-analysis are explained as a footnote.

\section{Results}

3.1. Identified Studies. The abstracts of 30 studies were assessed for eligibility. Twenty-one studies [22-42] were eliminated because they did not match the inclusion criteria or were not available in full text (Figure 1). A total of 9 studies were included in the quality assessment phase [43-51].

3.2. Quality Assessment of Study. The 9 included studies had an average PEDro score of 5.7/10, as illustrated in Table 1. Agreement between reviewers was good (unweighted $\kappa=$ 0.79 ). However, multiple sources of bias in these studies may have skewed the results. The most common shortcomings were lack of randomization [46-49, 51], lack of concealed allocation [46-49, 51], and lack of blinding (patient, therapist, or assessor) [43-51]. The most adhered ones to items on the PEDro scale were baseline comparability, follow-up, intention-to-treat analysis, measurements of variability, and between-group comparisons, which were evident in almost all the trials.

Agreement between the reviewers was excellent (unweighted $\kappa=0.87$ ) in assessing risk of bias across studies. Details of the risk of bias assessment of included studies are given in Table 2. The overall risk of bias assessment indicated that the risk of bias was low in 1 study [43], high in 5 studies $[46-49,51]$, and unclear in 3 studies $[44,45,50]$. The most common shortcomings were lack of blinding [47$49,51]$, lack of concealment [46-49, 51], and inadequate random sequence generation $[46-49,51]$.

3.3. Characteristics of Study Populations. Table 3 details participant characteristics. The sample size for whole study groups ranged from 30 to 252, with the mean age varying from 12 to 15 years. In most of the studies, the majority of participants with AIS were female [43, 44, 46-49, 51]. Most of the studies used the Cobb angle and Risser sign as inclusion criteria for participants with AIS [43-49, 51]. 


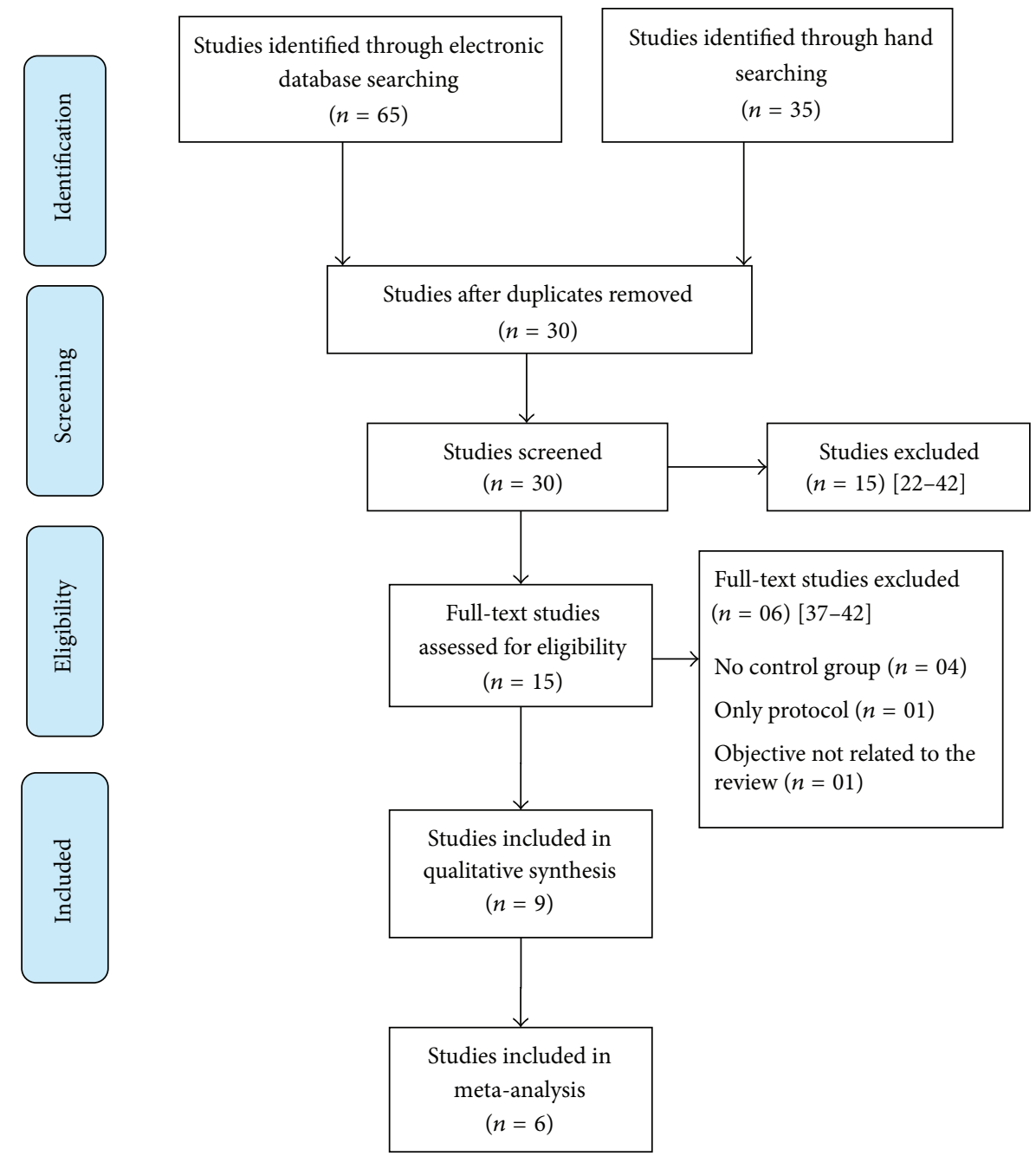

FIGURE 1: Flow diagram of the study procedure.

TABLE 1: Methodological classification assessed by PEDro scale.

\begin{tabular}{|c|c|c|c|c|c|c|c|c|c|c|}
\hline Criteria & $\begin{array}{l}\text { Monticone } \\
\text { et al. } \\
\text { (2014) [43] }\end{array}$ & $\begin{array}{c}\text { Kuru et } \\
\text { al. }(2015) \\
{[44]}\end{array}$ & $\begin{array}{c}\text { Diab } \\
(2012) \\
{[45]}\end{array}$ & $\begin{array}{c}\text { Noh et } \\
\text { al. (2014) } \\
{[46]}\end{array}$ & $\begin{array}{c}\text { Negrini et } \\
\text { al. (2006) } \\
{[47]}\end{array}$ & $\begin{array}{c}\text { Negrini et } \\
\text { al. (2006) } \\
{[48]}\end{array}$ & $\begin{array}{l}\text { Weiss and } \\
\text { Klein } \\
(2006) \\
{[49]}\end{array}$ & $\begin{array}{c}\text { Weiss et } \\
\text { al. }(2002) \\
{[50]}\end{array}$ & $\begin{array}{c}\text { Negrini et } \\
\text { al. (2008) } \\
{[51]}\end{array}$ & $\begin{array}{c}\text { Cumulative } \\
\text { score }^{*}\end{array}$ \\
\hline Random allocation? & Yes & Yes & Yes & No & No & No & No & Yes & No & 4 \\
\hline Concealed allocation? & Yes & Yes & Yes & No & No & No & No & Yes & No & 4 \\
\hline Baseline comparability? & Yes & Yes & Yes & Yes & Yes & Yes & Yes & No & Yes & 8 \\
\hline Blind participants? & Yes & No & No & No & No & No & No & No & No & 1 \\
\hline Blind therapists? & No & No & No & No & No & No & No & No & No & 0 \\
\hline Blind assessors? & Yes & No & No & Yes & No & No & No & No & No & 2 \\
\hline Follow-up? & Yes & Yes & Yes & Yes & Yes & Yes & Yes & Yes & Yes & 9 \\
\hline Intention-to-treat analysis? & Yes & Yes & Yes & Yes & Yes & Yes & Yes & Yes & Yes & 9 \\
\hline Group comparisons? & Yes & Yes & Yes & Yes & Yes & Yes & No & No & Yes & 7 \\
\hline Point and variability measures? & Yes & Yes & Yes & Yes & Yes & Yes & Yes & No & No & 7 \\
\hline Cumulative score & 9 & 7 & 7 & 6 & 5 & 5 & 4 & 4 & 4 & $5.7^{\dagger}$ \\
\hline
\end{tabular}

* Out of the 10 total studies.

${ }^{\dagger}$ Maximum score of 10 . 
TABLE 2: Risk of bias of included studies (yes, low risk of bias; no, high risk of bias).

\begin{tabular}{|c|c|c|c|c|c|c|}
\hline Citations & $\begin{array}{c}\text { Adequate sequence } \\
\text { generation? }\end{array}$ & $\begin{array}{c}\text { Allocation } \\
\text { concealment? }\end{array}$ & Blinding? & $\begin{array}{l}\text { Incomplete outcome } \\
\text { data addressed? }\end{array}$ & $\begin{array}{l}\text { Free of selective } \\
\text { reporting? }\end{array}$ & Conclusions \\
\hline Monticone et al. (2014) [43] & Yes & Yes & Yes & Yes & Yes & Low risk of bias \\
\hline Kuru et al. (2015) [44] & Yes & Yes & Unclear & Yes & Yes & Unclear risk of bias \\
\hline Diab (2012) [45] & Yes & Yes & Unclear & Yes & Yes & Unclear risk of bias \\
\hline Noh et al. (2014) [46] & No & No & Yes & Yes & Yes & High risk of bias \\
\hline Negrini et al. (2006) [47] & No & No & No & Yes & Yes & High risk of bias \\
\hline Negrini et al. (2006) [48] & No & No & No & Yes & Yes & High risk of bias \\
\hline Weiss and Klein (2006) [49] & No & No & No & Yes & Yes & High risk of bias \\
\hline Weiss et al. (2002) [50] & Yes & Yes & Unclear & Yes & Yes & Unclear risk of bias \\
\hline Negrini et al. (2008) [51] & No & No & No & Yes & Yes & High risk of bias \\
\hline
\end{tabular}

3.4. Training Protocol. Table 3 summarizes the training protocol. Three studies compared the Scientific Exercise Approach to Scoliosis (SEAS.02) exercises with controls [47, $48,51], 1$ study compared active self-correction and taskoriented exercises with controls [43], 1 study compared Schroth exercises with controls [44], 1 study compared forward head correction and traditional exercise with controls [45], 1 study compared the 3D corrective spinal technique with controls [46], 1 study compared physiologic exercise program and scoliosis intensive rehabilitation (SIR) with control [49], and 1 study compared passive transverse force and SIR with a control group [50]. In all included studies, the control group received usual care or performed a traditional exercise program. Only one study [43] had a report of an adverse effect which was a minor temporary worsening of pain.

3.5. Outcome Measures. Six studies used the Cobb angle [43, $44,46-48,51], 5$ studies used the angle of trunk rotation [43, 44, 47, 48, 51], 3 studies used the thoracic kyphosis angle $[45,46,49], 2$ studies used the lumbar lordosis angle $[45,46]$, and 3 studies used the average lateral deviation [45, $49,50]$ to measure various spinal deformities. Radiographic methods were used to measure the Cobb angle in all six included studies, and a Scoliometer was used to measure the angle of trunk rotation in the 5 included studies. Two studies used a Formetric device to measure thoracic kyphosis $[45,49]$, and 1 study used a radiographic method for this measurement [46]. One study used a Formetric device to measure lumbar lordosis [45], whereas the other study used a radiographic method for this measurement [46]. Average lateral deviation was measured with a Formetric device in all 3 included studies. Two studies used the Scoliosis Research Society-22 patient questionnaire (SRS-22) [43, 46], 1 study used SRS-23 [44], and another used the Functional Rating Index to measure health related quality of life [45]. The Functional Rating Index is a patient-rated scale composed of 10 items including 4 subscales: pain, sleep, work, and daily activity [52]. The subscales include 3 domains of the World Health Organization International Classification of Functioning, Disability, and Health (WHO-ICF) such as activity limitations with 6 items (personal care, travel, recreation, lifting, walking, and standing), impairment with 3 items (pain frequency, pain intensity, and sleep), and participation restriction with 1 item (work). Each item was scored on a 5point scale ranging from 0 (no pain or maximum ability) to 4 (maximum pain or disability). The possible score ranges from $0 \%$ (no disability) to $100 \%$ (severe disability).

3.6. Effect of Exercise on Spinal Deformities. Table 4 gives details of the results of the exercise and control group in included studies. Data syntheses of included studies are given in Table 5 and Figures 2-7. Meta-analysis of 4 studies [43, 44, $46,47]$ provided moderate-quality evidence with a significant effect size point estimate across the 4 included studies $(p=$ 0.000 ), with an overall medium effect size point estimate of 0.65 ( $95 \%$ CI, -0.89 to -0.40$)$ based on a fixed-effects model that favored exercise compared with controls in reducing the Cobb angle (Figure 2).

Meta-analysis of 2 studies [43, 44] provided moderatequality evidence with a significant effect $(p=0.000)$, with an overall medium effect size point estimate of 0.73 (95\% CI, -1.07 to -0.39 ) based on a fixed-effects model that favored exercise compared with controls in reducing the angle of trunk rotation (Figure 3).

Meta-analysis of 3 studies $[45,46,49]$ provided moderatequality evidence with a significant effect size point estimate across the 3 included studies $(p=0.001)$, with an overall medium effect size point estimate of 0.55 ( $95 \% \mathrm{CI},-0.89$ to -0.22 ) based on a fixed-effects model that favored exercise compared with controls in reducing the thoracic kyphosis angle (Figure 4 ).

Meta-analysis of 2 studies $[45,46]$ provided moderatequality evidence with a significant overall effect ( $p=0.003$ ), with an overall medium effect size point estimate of $0.57(95 \%$ CI, -0.96 to -0.19 ) based on a fixed-effects model that favored exercise compared with controls in reducing lumbar lordosis (Figure 5).

Meta-analysis of 2 studies $[45,50]$ provided low-quality evidence with a significant overall effect $(p=0.005)$, with an overall medium effect size point estimate of 0.54 (95\% CI, -0.92 to -0.16$)$ based on a fixed-effects model that favored 


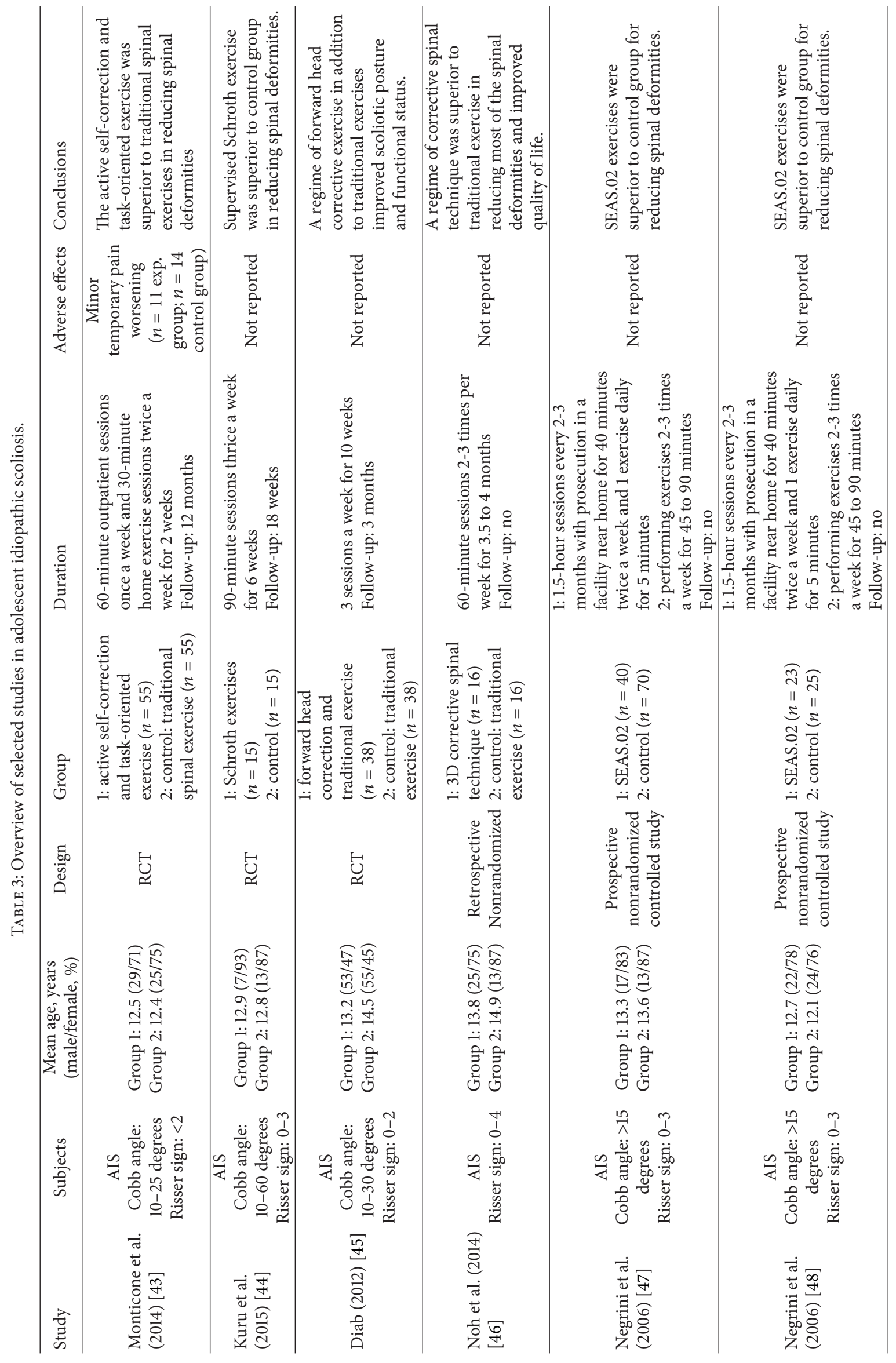




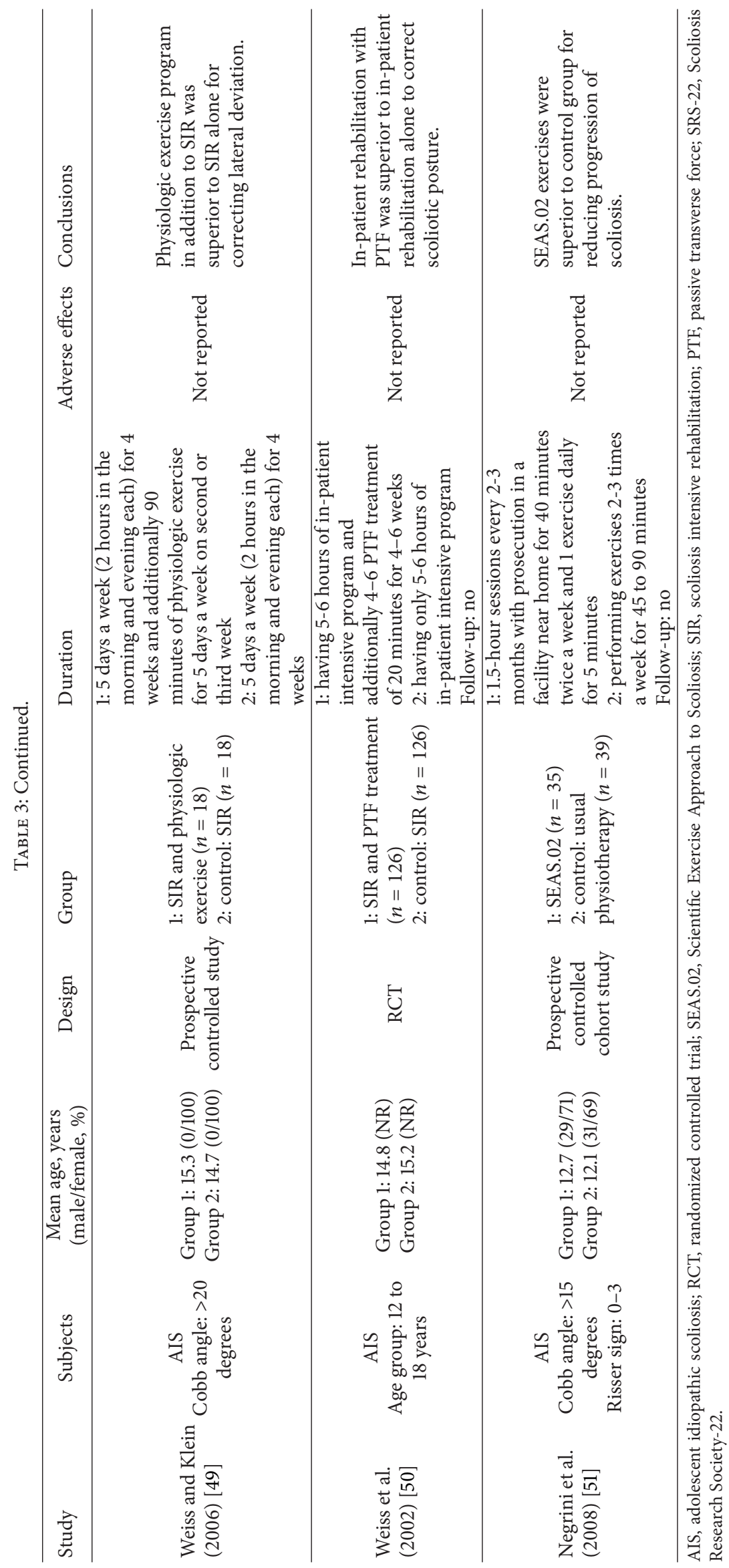




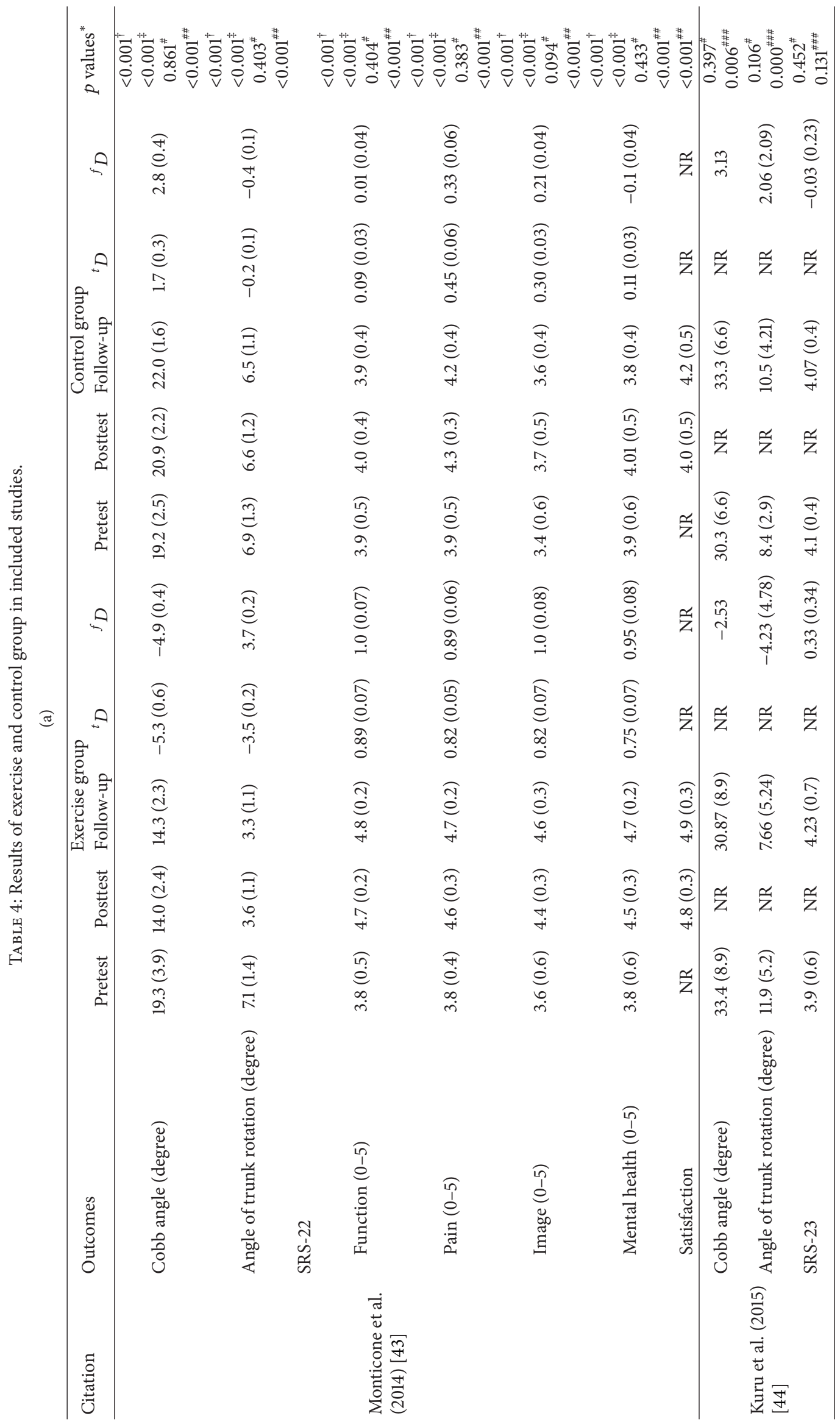




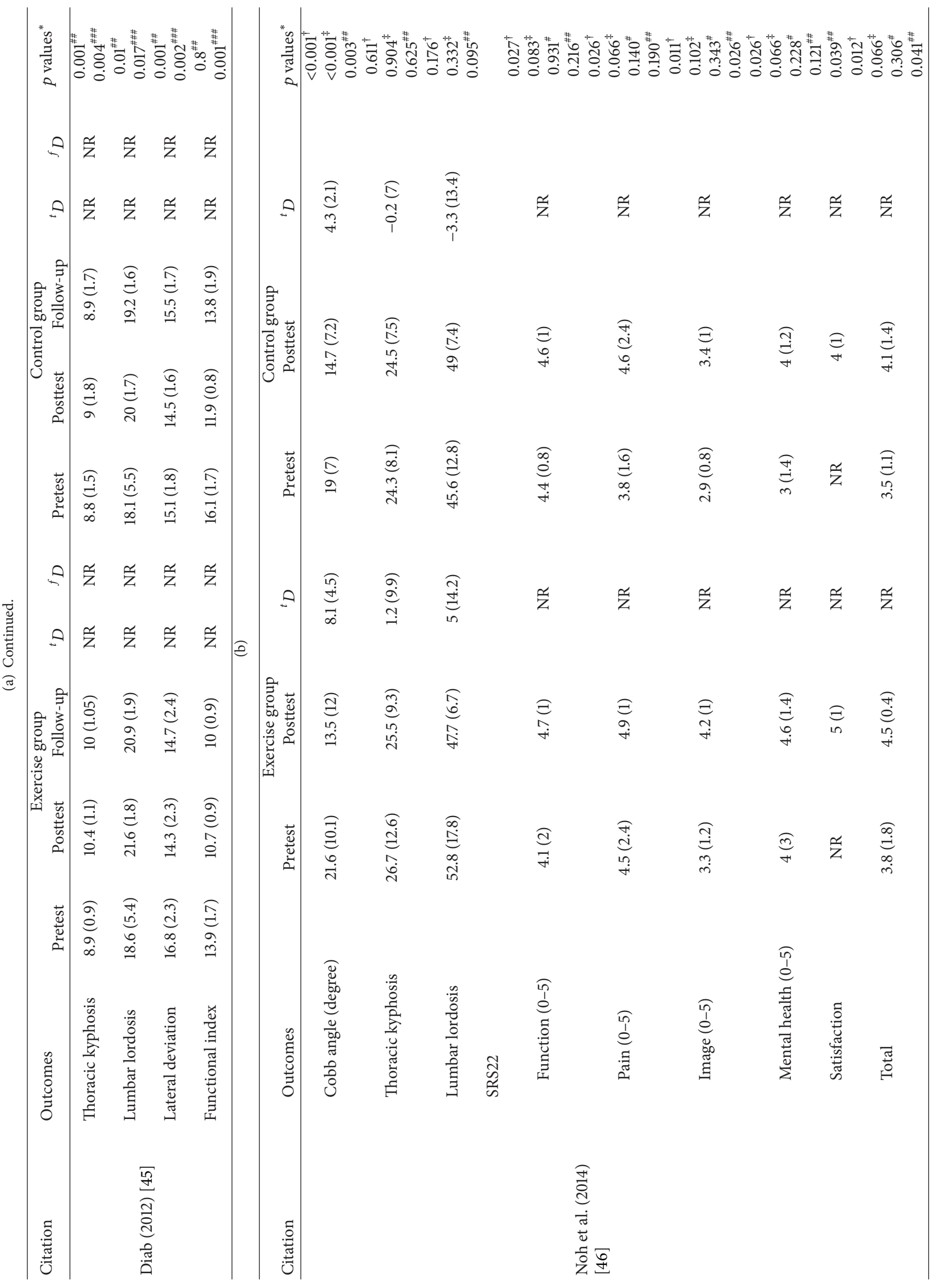




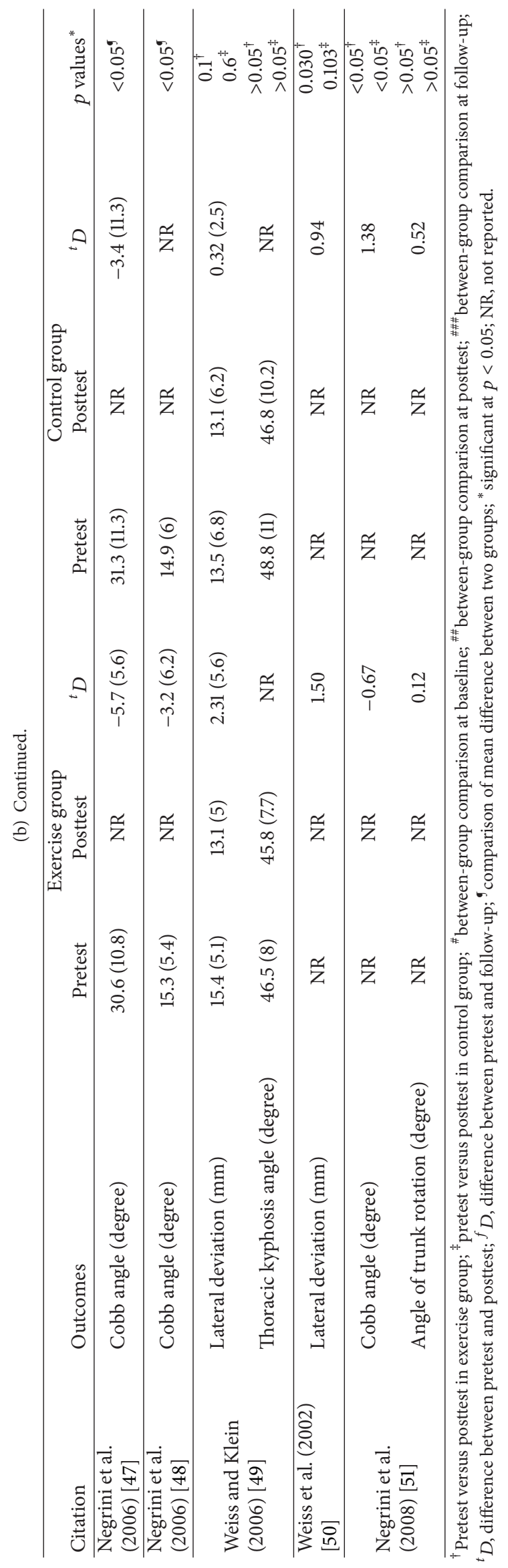


TABLE 5: Meta-analyses of effect of exercise program.

\begin{tabular}{lcccccc}
\hline Outcomes & Number of studies & $\begin{array}{c}\text { Ratio of studies } \\
\text { (PEDro }<6)\end{array}$ & $\begin{array}{c}\text { Number of } \\
\text { subjects }\end{array}$ & SMD [95\% CI $]$ & $\begin{array}{c}I^{2} \\
\text { Quality of evidence } \\
\text { (GRADE) }\end{array}$ \\
\hline Cobb angle & 4 & $25 \%$ & 282 & $0.65[-0.89,-0.40]$ & $30.53 \%$ & Moderate $^{\dagger}$ \\
Angle of trunk rotation & 2 & $0 \%$ & 140 & $0.73[-1.07,-0.39]$ & $1.49 \%$ & Moderate $^{\ddagger}$ \\
Thoracic kyphosis angle & 3 & $33 \%$ & 144 & $0.55[-0.89,-.22]$ & $0 \%$ & Moderate $^{\ddagger}$ \\
Lumbar lordosis angle & 2 & $0 \%$ & 108 & $0.57[-0.96,-0.19]$ & $0 \%$ & Moderate $^{\ddagger}$ \\
Average lateral deviation & 2 & $50 \%$ & 112 & $0.54[-0.92,-0.16]$ & $46 \%$ & Low $^{5}$ \\
Quality of life & 3 & $0 \%$ & 138 & $0.73[-1.07,-0.38]$ & $0 \%$ & Moderate $^{\ddagger}$ \\
\hline
\end{tabular}

GRADE, GRADE working group grades of evidence.

${ }^{\dagger}$ Statistical heterogeneity results downgrade $\left(I^{2}>25 \%\right)$. ${ }^{\ddagger}$ Large confidence interval results downgrade. ${ }^{9}$ Large confidence interval, statistical heterogeneity results downgrade.

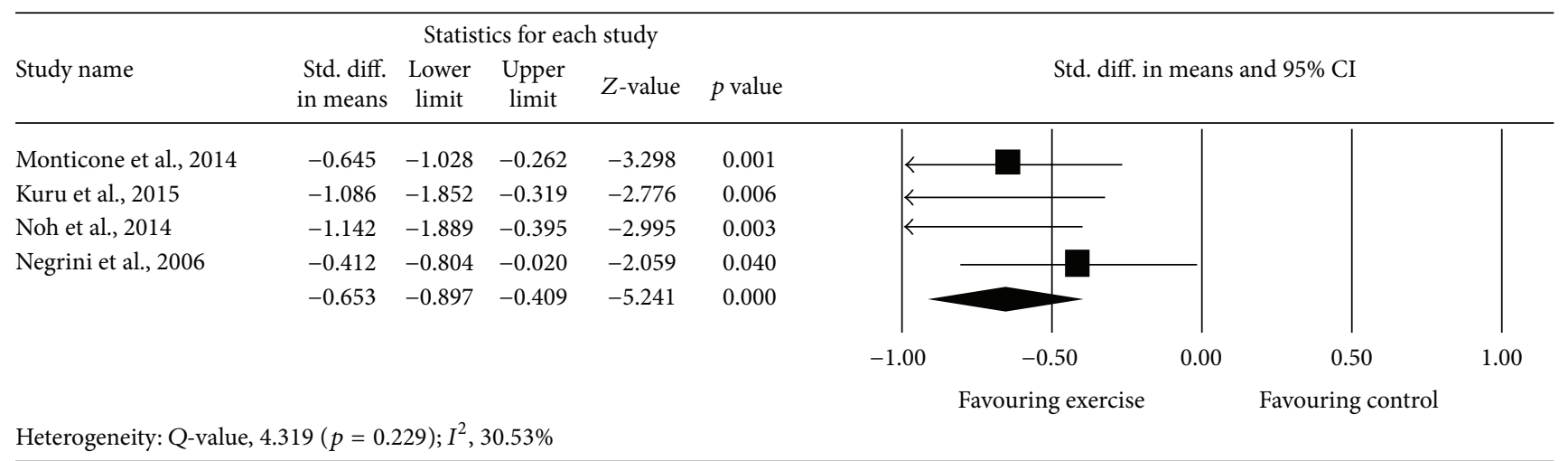

FIGURE 2: Effect of exercise on the Cobb angle.

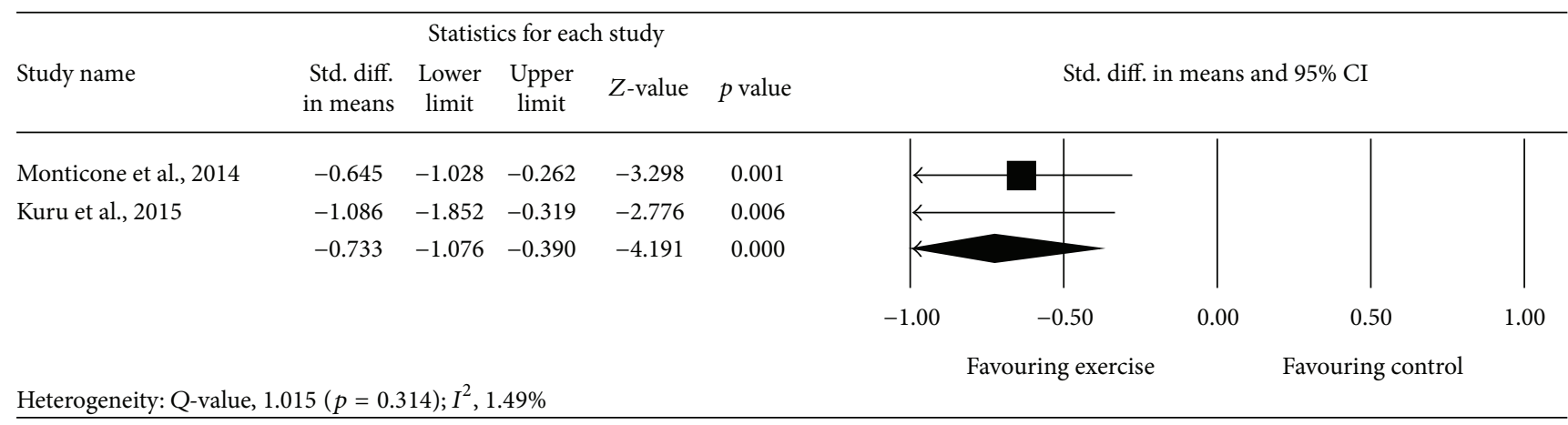

FIGURE 3: Effect of exercise on the angle of trunk rotation.

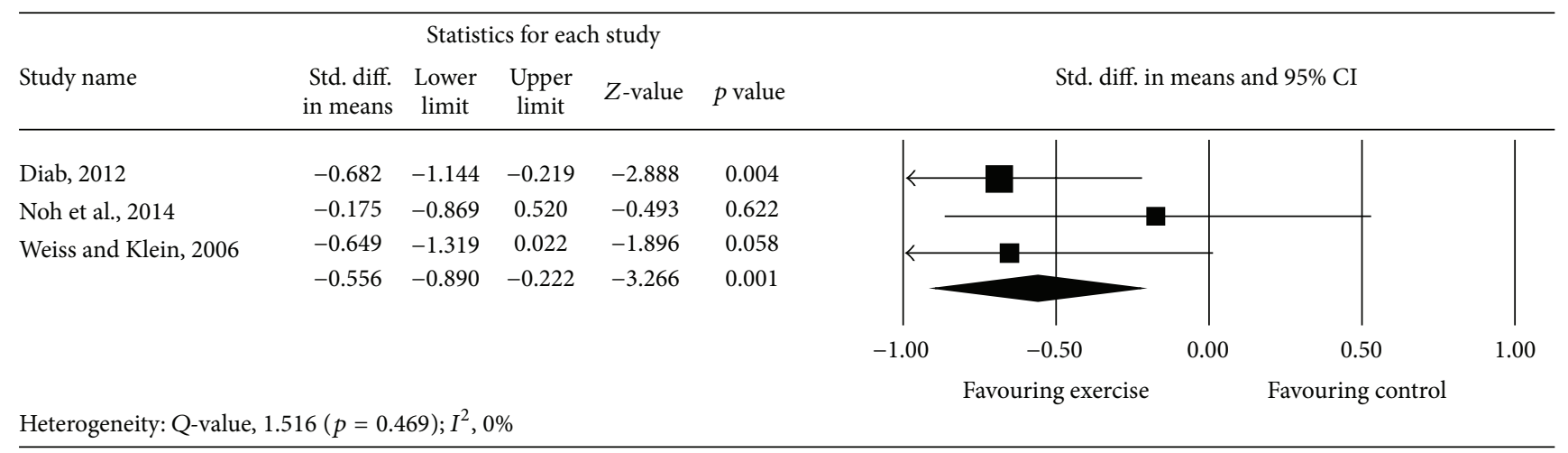

FIGURE 4: Effect of exercise on the thoracic kyphosis angle. 


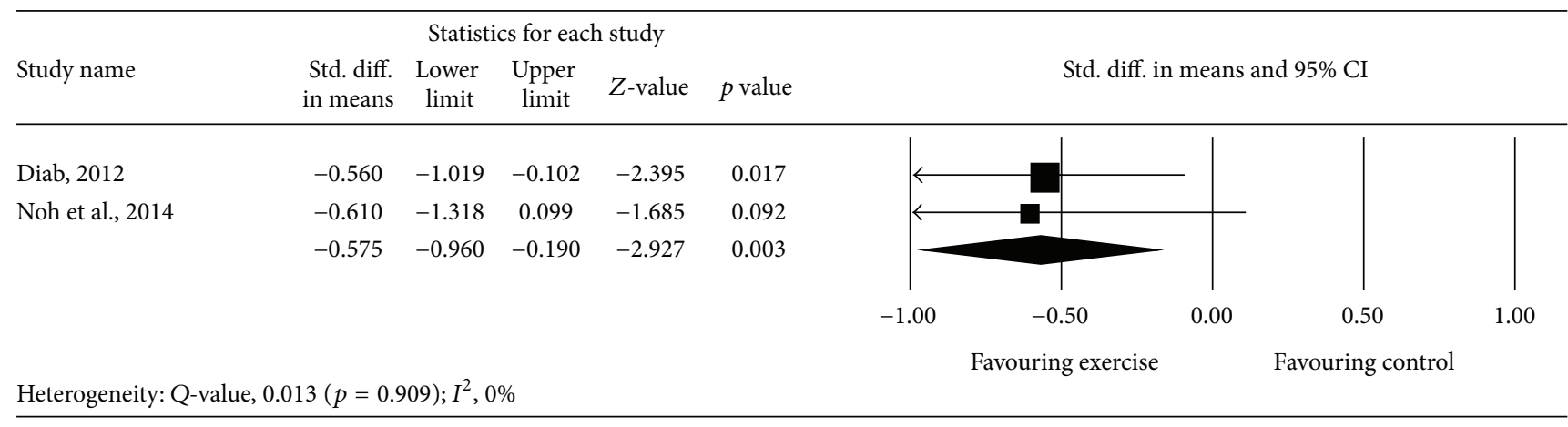

FIgURE 5: Effect of exercise on the lumbar lordosis angle.

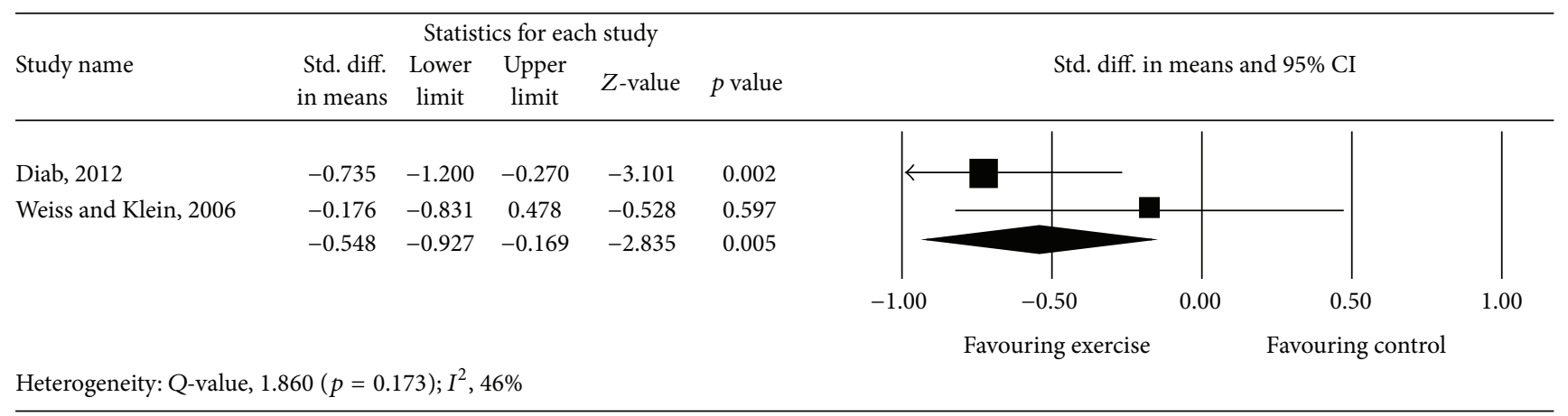

FIGURE 6: Effect of exercise on the average lateral deviation.

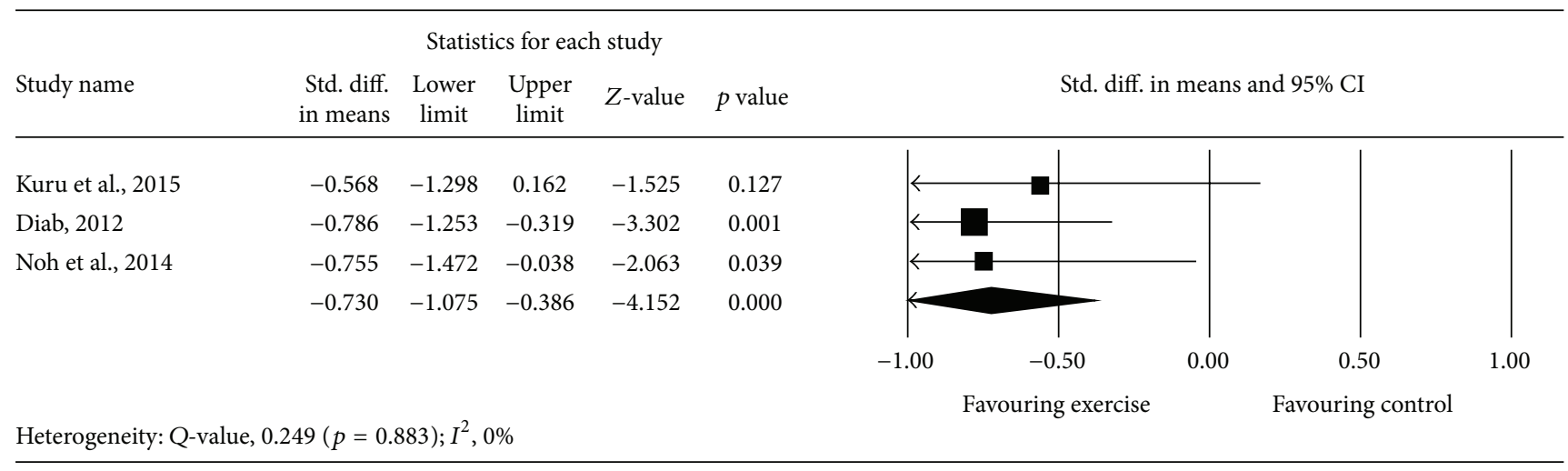

FIGURE 7: Effect of exercise on the quality of life.

exercise compared with controls in reducing average lateral deviation (Figure 6).

3.7. Effect of Exercise on Quality of Life. Meta-analysis of 3 studies [44-46] provided moderate-quality evidence with a significant effect size point estimate across the 3 included studies $(p=0.000)$, with an overall medium effect size point estimate of 0.73 (95\% CI, -1.07 to -0.03 ) based on a fixedeffects model that favored exercise compared with controls in improving the quality of life (Table 5 and Figure 7).

\section{Discussion}

This systematic review investigated current available evidence on the effects of an exercise program on spinal deformities 
and quality of life in patients with AIS. The review evaluated 9 studies, including a total of 768 participants.

Among the 9 studies evaluated using the PEDro scale [17], 6 were considered of high methodological quality [43-48]. The overall risk of bias assessment showed that 5 studies had a high risk of bias [46-49, 51], and 1 study had a low risk of bias [43], while others had an unclear risk of bias $[44,45,50]$. More than half of the studies failed to perform blinding and (Table 2).

The results of the present systematic review provide moderate-quality evidence for exercise intervention with a medium effect size for reducing the Cobb angle, angle of trunk rotation, thoracic kyphosis angle, and lumbar lordosis angle and improving the quality of life in patients with AIS. Similarly, a systematic review conducted by Fusco et al. [53] reported improvement in the Cobb angle following a regime of exercise. In another review, Negrini et al. [10] confirmed the efficacy of exercises in reducing the progression of deformity and Cobb angles in patients with AIS. In contrast, Mordecai and Dabke [8] reported poor quality evidence supporting the use of an exercise program in the management of AIS, and a Cochrane review conducted by Romano et al. [12] revealed a lack of high-quality evidence to recommend the use of scoliosis-specific exercises to reduce the progression of AIS.

All previous reviews were focused on the effects of exercise on the Cobb angle and brace prescriptions in patients with AIS [8, 10, 53]. However, in the present review, in addition to the Cobb angle, other surface spinal deformities such as trunk rotation, thoracic kyphosis, lumbar lordosis, average lateral deviation, and quality of life were measured. Moreover, in previous reviews, only Romano et al. [12] performed a meta-analysis of the effects of scoliosis-specific exercises to reduce the progression of AIS.

In the present review, 3 studies compared SEAS.02 exercise with a control group and reported that SEAS.02 exercises were superior to control conditions for reducing spinal deformities and the progression of scoliosis $[47,48,51]$. Another 6 studies included in the present review compared 6 different exercise protocols with traditional spinal exercises. All these studies reported significant reduction of spinal deformities and improvement in quality of life as compared with traditional spinal exercise [43-46, 49, 50].

This review had several limitations. Inclusion criteria were not well defined in the included studies, and the majority of the included studies were nonrandomized. Additionally, lack of blinding, lack of concealed allocation, and variations in exercise protocols are significant limitations in the current published literature. Moreover, different types of exercise have different intensities and may induce different effects, and the presence of heterogeneity in exercise protocols prevents conclusive results. For example, the total intervention duration varied between 2 weeks [43] and 4 months [46] and sample size in the included studies varied from 30 [44] to 252 [50]. Another limitation of the present review was the inclusion of only studies published in English, which might have created some selection bias. In addition, most of the included studies did not clarify what types of exercises are found in the usual care.

\section{Conclusions}

Moderate-quality evidence suggests that an exercise program is superior to controls in reducing the Cobb angle, angle of trunk rotation, thoracic kyphosis angle, and lumbar lordosis angle and improving the quality of life in patients with AIS; and the low-quality evidence suggests that an exercise program is superior to controls in reducing average lateral deviation in patients with AIS. However, the presence of heterogeneity in exercise protocols and poor methodological quality limit the validity of these results.

\section{Conflict of Interests}

The authors declared that there is no conflict of interests regarding the publication of this paper.

\section{Acknowledgment}

The project was full financially supported by King Saud University, through Vice Deanship of Research Chairs, Rehabilitation Research Chair.

\section{References}

[1] M. C. Hawes and J. P. O'Brien, “The transformation of spinal curvature into spinal deformity: pathological processes and implications for treatment," Scoliosis, vol. 1, no. 1, article 3, 2006.

[2] M. A. Asher and D. C. Burton, "Adolescent idiopathic scoliosis: natural history and long term treatment effects," Scoliosis, vol. 1, article 2, 2006.

[3] S. L. Weinstein, L. A. Dolan, J. C. Cheng, A. Danielsson, and J. A. Morcuende, "Adolescent idiopathic scoliosis," The Lancet, vol. 371, no. 9623, pp. 1527-1537, 2008.

[4] S. L. Weinstein and I. V. Ponseti, "Curve progression in idiopathic scoliosis," The Journal of Bone \& Joint Surgery Series A, vol. 65, no. 4, pp. 447-455, 1983.

[5] E. Ascani, P. Bartolozzi, C. A. Logroscino et al., "Natural history of untreated idiopathic scoliosis after skeletal maturity," Spine, vol. 11, no. 8, pp. 784-789, 1986.

[6] M. S. Goldberg, N. E. Mayo, B. Poitras, S. Scott, and J. Hanley, "The Ste-Justine adolescent idiopathic scoliosis cohort study. Part II. Perception of health, self and body image, and participation in physical activities," Spine, vol. 19, no. 14, pp. 1562-1572, 1994.

[7] M. S. Wong, J. C. Y. Cheng, T. P. Lam et al., "The effect of rigid versus flexible spinal orthosis on the clinical efficacy and acceptance of the patients with adolescent idiopathic scoliosis," Spine, vol. 33, no. 12, pp. 1360-1365, 2008.

[8] S. C. Mordecai and H. V. Dabke, "Efficacy of exercise therapy for the treatment of adolescent idiopathic scoliosis: a review of the literature," European Spine Journal, vol. 21, no. 3, pp. 382-389, 2012.

[9] S. L. Weinstein, L. A. Dolan, J. G. Wright, and M. B. Dobbs, "Effects of bracing in adolescents with idiopathic scoliosis," The New England Journal of Medicine, vol. 369, no. 16, pp. 1512-1521, 2013. 
[10] S. Negrini, C. Fusco, S. Minozzi, S. Atanasio, F. Zaina, and M. Romano, "Exercises reduce the progression rate of adolescent idiopathic scoliosis: results of a comprehensive systematic review of the literature," Disability and Rehabilitation, vol. 30, no. 10, pp. 772-785, 2008.

[11] S. Negrini, J.-C. De Mauroy, T. B. Grivas et al., "Actual evidence in the medical approach to adolescents with idiopathic scoliosis," European Journal of Physical and Rehabilitation Medicine, vol. 50, no. 1, pp. 87-92, 2014.

[12] M. Romano, S. Minozzi, F. Zaina et al., "Exercises for adolescent idiopathic scoliosis," Spine, vol. 38, no. 14, pp. E883-E893, 2013.

[13] S. Negrini, G. Antonini, R. Carabalona, and S. Minozzi, "Physical exercises as a treatment for adolescent idiopathic scoliosis. A systematic review," Pediatric Rehabilitation, vol. 6, no. 3-4, pp. 227-235, 2003.

[14] J. Higgins and S. Green, Cochrane Handbook for Systematic Reviews of Interventions Version 5.0.0, The Cochrane Collaboration, Chichester, UK, 2008.

[15] J. Cohen, "Quantitative methods in psychology," Psychological Bulletin, vol. 112, pp. 155-159, 1992.

[16] Biostat, Comprehensive Meta-Analysis Version 2.0, [Computer software], Biostat, Englewood, NJ, USA, 2014.

[17] C. G. Maher, C. Sherrington, R. D. Herbert, A. M. Moseley, and M. Elkins, "Reliability of the PEDro scale for rating quality of randomized controlled trials," Physical Therapy, vol. 83, no. 8, pp. 713-721, 2003.

[18] J. A. Wallis and N. F. Taylor, "Pre-operative interventions (nonsurgical and non-pharmacological) for patients with hip or knee osteoarthritis awaiting joint replacement surgery-a systematic review and meta-analysis," Osteoarthritis and Cartilage, vol. 19, no. 12, pp. 1381-1395, 2011.

[19] J. P. T. Higgins and D. G. Altman, "Assessing risk of bias in included studies," in Cochrane Handbook for Systematic Reviews of Interventions Version 5.0.1, J. P. T. Higgins and S. Green, Eds., The Cochrane Collaboration, Oxford, UK, 2008.

[20] D. Atkins, D. Best, P. A. Briss et al., "Grading quality of evidence and strength of recommendations," The British Medical Journal, vol. 328, no. 7454, p. 1490, 2004.

[21] J. P. T. Higgins, S. G. Thompson, J. J. Deeks, and D. G. Altman, "Measuring inconsistency in meta-analyses," British Medical Journal, vol. 327, no. 7414, pp. 557-560, 2003.

[22] B. Falk, W. A. Rigby, and N. Akseer, "Adolescent idiopathic scoliosis: the possible harm of bracing and the likely benefit of exercise," Spine Journal, vol. 15, no. 1, pp. 209-210, 2015.

[23] M. Płaszewski, I. Cieśliński, P. Kowalski, A. Truszczyńska, and R. Nowobilski, "Does scoliosis-specific exercise treatment in adolescence alter adult quality of life?" The Scientific World Journal, vol. 2014, Article ID 539671, 10 pages, 2014.

[24] K. Fabian and K. Rożek-Piechura, "Exercise tolerance and selected motor skills in young females with idiopathic scoliosis treated with different physiotherapeutic methods," Ortopedia Traumatologia Rehabilitacja, vol. 16, no. 5, pp. 507-522, 2014.

[25] V. L. dos Santos Alves, R. J. Alves da Silva, and O. Avanzi, "Effect of a preoperative protocol of aerobic physical therapy on the quality of life of patients with adolescent idiopathic scoliosis: a randomized clinical study," The American Journal of Orthopedics (Belle Mead, NJ), vol. 43, pp. E112-E116, 2014.

[26] N. Pugacheva, "Corrective exercises in multimodality therapy of idiopathic scoliosis in children-analysis of six weeks efficiency-pilot study," Studies in Health Technology and Informatics, vol. 176, pp. 365-371, 2012.
[27] D. Czaprowski, T. Kotwicki, R. Biernat, J. Urniaz, and A. Ronikier, "Physical capacity of girls with mild and moderate idiopathic scoliosis: influence of the size, length and number of curvatures," European Spine Journal, vol. 21, no. 6, pp. 1099-1105, 2012.

[28] M. W. Morningstar, "Outcomes for adult scoliosis patients receiving chiropractic rehabilitation: a 24-month retrospective analysis," Journal of Chiropractic Medicine, vol. 10, no. 3, pp. 179184, 2011.

[29] F. Canavese and A. Kaelin, "Adolescent idiopathic scoliosis: indications and efficacy of nonoperative treatment," Indian Journal of Orthopaedics, vol. 45, no. 1, pp. 7-14, 2011.

[30] Y. T. Tsai, C.-P. Leong, Y.-C. Huang et al., "The electromyographic responses of paraspinal muscles during isokinetic exercise in adolescents with idiopathic scoliosis with a Cobb's angle less than fifty degrees," Chang Gung Medical Journal, vol. 33, no. 5, pp. 540-550, 2010.

[31] S. D. Glassman, L. Y. Carreon, C. I. Shaffrey et al., "The costs and benefits of nonoperative management for adult scoliosis," Spine, vol. 35, no. 5, pp. 578-582, 2010.

[32] M.-L. B. Lenssinck, A. C. Frijlirik, M. Y. Berger, S. M. A. BiermaZeinstra, K. Verkerk, and A. P. Verhagen, "Effect of bracing and other conservative interventions in the treatment of idiopathic scoliosis in adolescents: a systematic review of clinical trials," Physical Therapy, vol. 85, no. 12, pp. 1329-1339, 2005.

[33] K. Ferrari, P. Goti, A. Sanna et al., "Short-term effects of bracing on exercise performance in mild idiopathic thoracic scoliosis," Lung, vol. 175, no. 5, pp. 299-310, 1997.

[34] H. R. Weiss, "The effect of an exercise program on vital capacity and rib mobility in patients with idiopathic scoliosis," Spine, vol. 16, no. 1, pp. 88-93, 1991.

[35] J. M. Shneerson and R. Madgwick, "the effect of physical training on exercise ability in adolescent idiopathic scoliosis," Acta Orthopaedica, vol. 50, no. 3, pp. 303-306, 1979.

[36] A. L. Nachemson, J. C. Bjure, L. G. Grimby, and M. Lindh, "Physical fitness in young women with idiopathic scoliosis before and after an exercise program," Archives of Physical Medicine and Rehabilitation, vol. 51, no. 2, pp. 95-98, 1970.

[37] S. Schreiber, E. C. Parent, D. M. Hedden, M. Moreau, D. Hill, and E. Lou, "Effect of Schroth exercises on curve characteristics and clinical outcomes in adolescent idiopathic scoliosis: protocol for a multicentre randomised controlled trial," Journal of Physiotherapy, vol. 60, no. 4, p. 234, 2014.

[38] H. R. Weiss and G. Weiss, "Curvature progression in patients treated with scoliosis in-patient rehabilitation-a sex and age matched controlled study," Studies in Health Technology and Informatics, vol. 91, pp. 352-356, 2002.

[39] S. Negrini, S. Donzelli, M. Lusini, S. Minnella, and F. Zaina, "The effectiveness of combined bracing and exercise in adolescent idiopathic scoliosis based on SRS and SOSORT criteria: a prospective study," BMC Musculoskeletal Disorders, vol. 15, article 263, 2014.

[40] C. Lewis, R. Diaz, G. Lopez, N. Marki, and B. Olivio, "A preliminary study to evaluate postural improvement in subjects with scoliosis: active therapeutic movement version 2 device and home exercises using the Mulligan's mobilization-withmovement concept," Journal of Manipulative and Physiological Therapeutics, vol. 37, pp. 502-509, 2014.

[41] L. Rivett, A. Stewart, and J. Potterton, "The effect of compliance to a Rigo System Cheneau brace and a specific exercise programme on idiopathic scoliosis curvature: a comparative study: SOSORT 2014 award winner," Scoliosis, vol. 9, article 5, 2014. 
[42] H. Hundozi-Hysenaj, I. B. Dallku, A. Murtezani, and S. Rrecaj, "Treatment of the idiopathic scoliosis with brace and physiotherapy," Nigerian Journal of Medicine, vol. 18, no. 3, pp. 256-259, 2009.

[43] M. Monticone, E. Ambrosini, D. Cazzaniga, B. Rocca, and S. Ferrante, "Active self-correction and task-oriented exercises reduce spinal deformity and improve quality of life in subjects with mild adolescent idiopathic scoliosis. Results of a randomised controlled trial," European Spine Journal, vol. 23, no. 6, pp. 1204-1214, 2014.

[44] T. Kuru, İ. Yeldan, E. E. Dereli, A. R. Özdinçler, F. Dikici, and İ. Colak, "The efficacy of three-dimensional Schroth exercises in adolescent idiopathic scoliosis: a randomised controlled clinical trial," Clinical Rehabilitation, 2015.

[45] A. A. Diab, "The role of forward head correction in management of adolescent idiopathic scoliotic patients: a randomized controlled trial," Clinical Rehabilitation, vol. 26, no. 12, pp. 11231132, 2012.

[46] D. K. Noh, J. S. You, J. H. Koh et al., "Effects of novel corrective spinal technique on adolescent idiopathic scoliosis as assessed by radiographic imaging," Journal of Back and Musculoskeletal Rehabilitation, vol. 27, pp. 331-338, 2014.

[47] S. Negrini, A. Negrini, M. Romano, N. Verzini, A. Negrini, and S. Parzini, "A controlled prospective study on the efficacy of SEAS.02 exercises in preparation to bracing for idiopathic scoliosis," Studies in Health Technology and Informatics, vol. 123, pp. 519-522, 2006.

[48] S. Negrini, A. Negrini, M. Romano, N. Verzini, A. Negrini, and S. Parzini, "A controlled prospective study on the efficacy of SEAS.02 exercises in preventing progression and bracing in mild idiopathic scoliosis," Studies in Health Technology and Informatics, vol. 123, pp. 523-526, 2006.

[49] H.-R. Weiss and R. Klein, "Improving excellence in scoliosis rehabilitation: a controlled study of matched pairs," Pediatric Rehabilitation, vol. 9, no. 3, pp. 190-200, 2006.

[50] H. R. Weiss, I. Heckel, and C. Stephan, "Application of passive transverse forces in the rehabilitation of spinal deformities: a randomized controlled study," Studies in Health Technology and Informatics, vol. 88, pp. 304-308, 2002.

[51] S. Negrini, F. Zaina, M. Romano, A. Negrini, and S. Parzini, "Specific exercises reduce brace prescription in adolescent idiopathic scoliosis: a prospective controlled cohort study with worst-case analysis," Journal of Rehabilitation Medicine, vol. 40, no. 6, pp. 451-455, 2008.

[52] R. J. Feise and J. M. Menke, "Functional rating index: literature review," Medical Science Monitor, vol. 16, no. 2, pp. RA25-RA36, 2010.

[53] C. Fusco, F. Zaina, S. Atanasio, M. Romano, A. Negrini, and S. Negrini, "Physical exercises in the treatment of adolescent idiopathic scoliosis: an updated systematic review," Physiotherapy Theory and Practice, vol. 27, no. 1, pp. 80-114, 2011. 


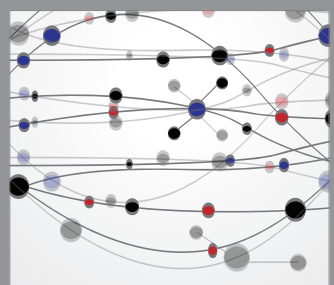

The Scientific World Journal
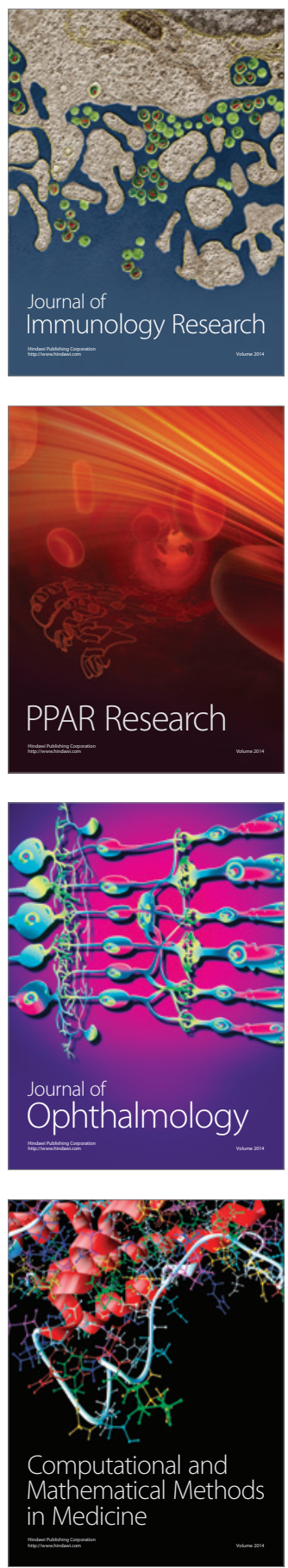

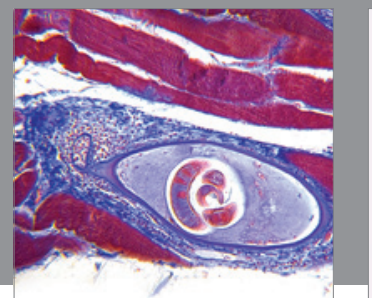

Gastroenterology

Research and Practice
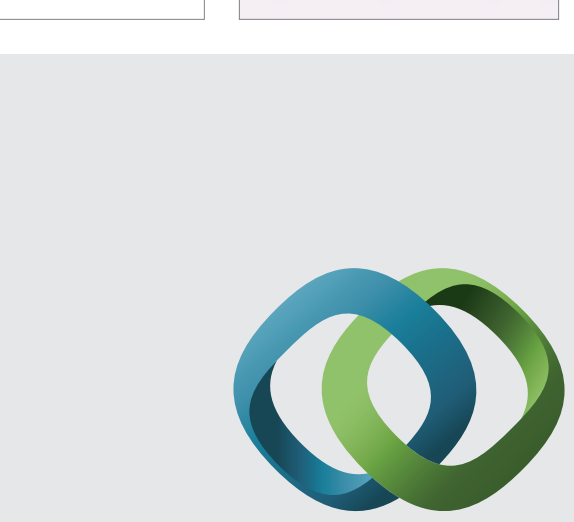

\section{Hindawi}

Submit your manuscripts at

http://www.hindawi.com
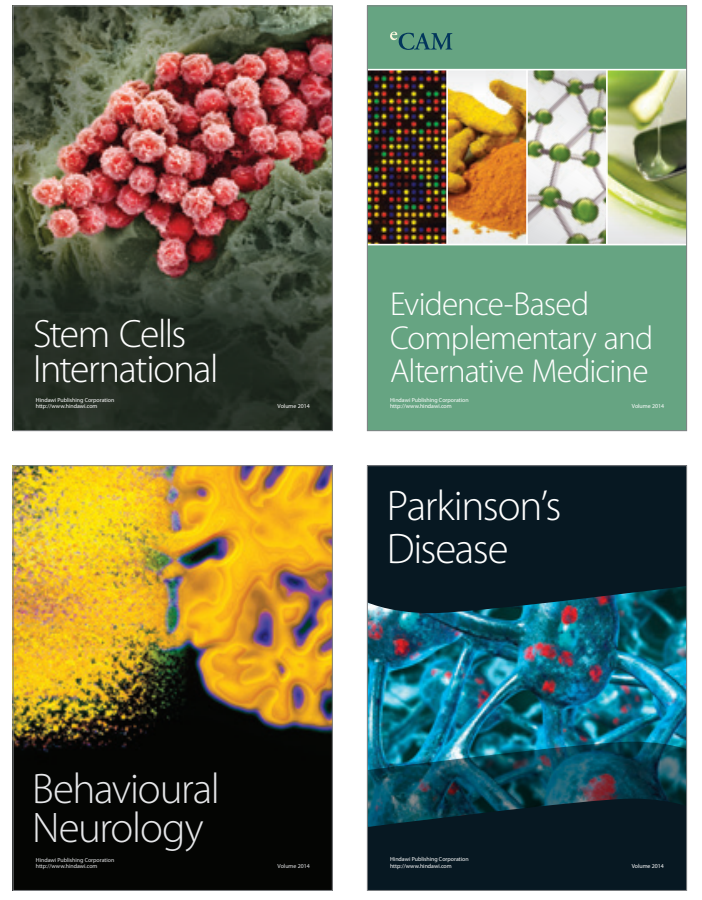
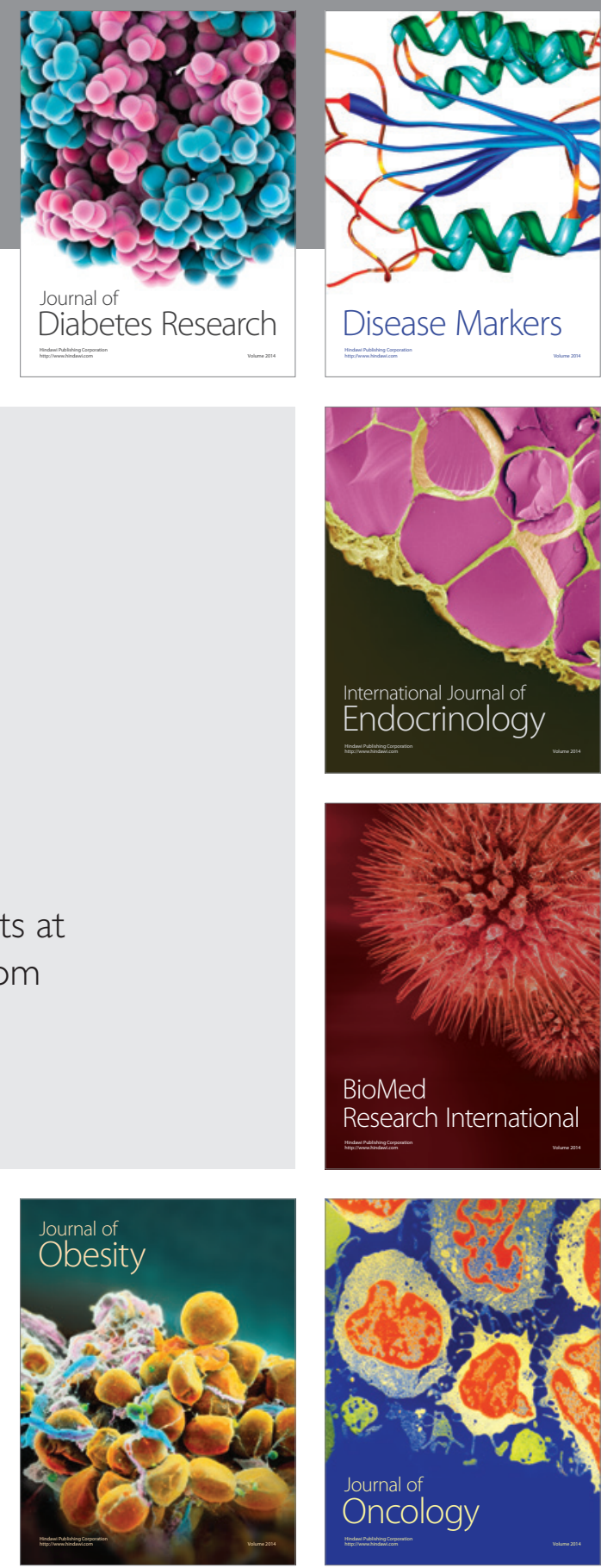

Disease Markers
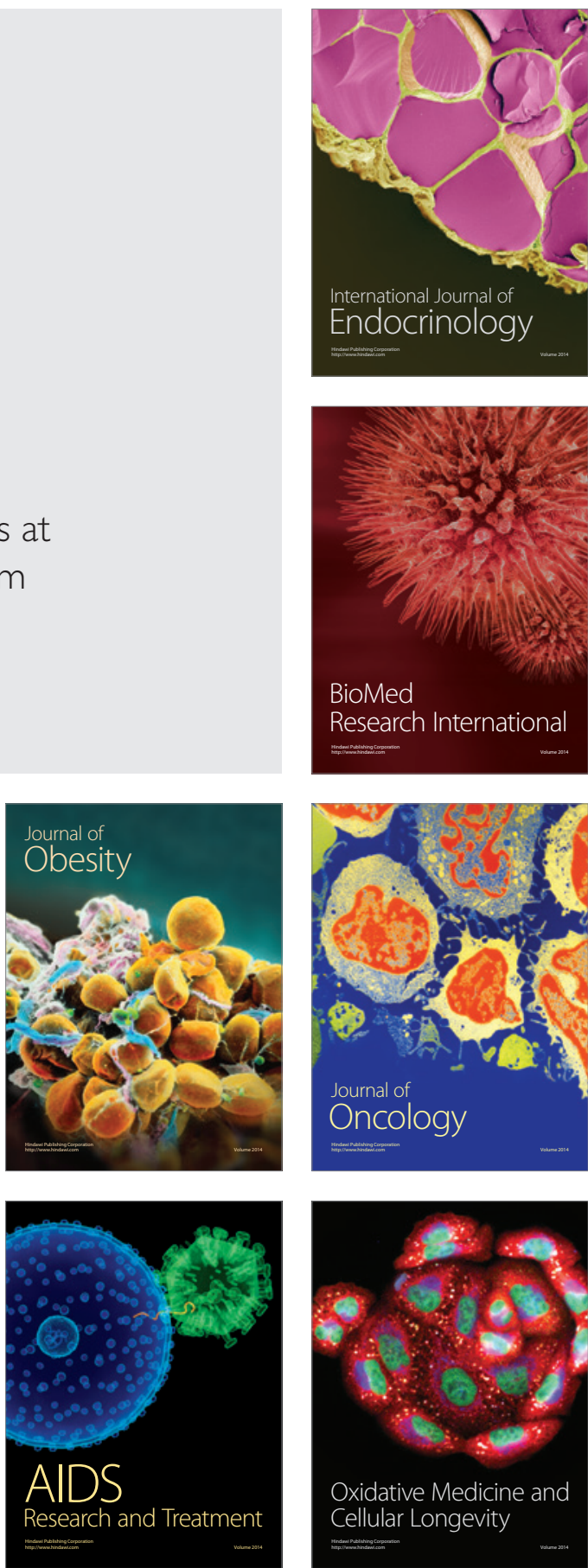